for all $g^{\prime} \in G$ either $S g^{\prime} \subseteq S$ or $S \subseteq S g^{\prime}$.

(6) There exists a family $\Sigma$ of subsets of $G$ such that $e=\bigcap_{s \in \Sigma} S$ and for all $g \in G$ and $S \in \Sigma$ either $S g \subseteq S$ or $S \subseteq S g$.

\title{
REFERENCES
}

1. P. Conrad, Right-ordered groups, Michigan Math. J. 6 (1959), 267-275.

2. M. Ohnishi, Linear-order on a group, Osaka Math. J. 4 (1952), 17-18.

California Institute of Technology

\section{NONEXTENDIBILITY CONDITIONS ON MUTUALLY ORTHOGONAL LATIN SQUARES}

\section{E. T. PARKER}

In this note are proved two theorems which impose restrictions on the structure of sets of mutually orthogonal latin squares. The corollary yields a version stronger in a certain direction than a known theorem on possible orders of subplanes of finite projective planes. This work developed from study of the first pairs of orthogonal latin squares of order 10, all of which had a pair of orthogonal latin subsquares of order $3([1 ; 2$, pp. 198-200]; another unpublished construction was found by Bose and Shrikhande). There exist pairs of orthogonal latin squares of order 10 having no pair of orthogonal latin subsquares of order 3 ; examples were generated with UNIVAC M-460 Computer, programmed by the author [3]. The arguments in this note depend on inequalities. A theorem of Mann $[4 ; 5]$ on orthogonal latin squares of orders $4 t+2$ was proved using inequalities and parities. The author feels that some combination of his own and Mann's methods might lead to better theorems.

THEOREM 1. If a set of $t$ mutually orthogonal latin squares (m.o.l.s.) of order $n$ has a set of $t$ mutually orthogonal latin subsquares (m.o.l. subs.) of order $r$, with $r<n$, then $n \geqq(t+1) r$.

Proof. Partition the rows and columns of the m.o.l.s. into sets of $n-r$ and $r$, with the m.o.l. subs. contained in the sets of $r$ rows and $r$ columns. Designate the $(n-r) \times(n-r)$ block by $A$, the $r \times r$ block by $D$, and the two rectangular blocks by $B$ and $C$. Let $x_{1}, \cdots, x_{r}$

Presented to the Society, January 28,1960 ; received by the editors January 17 , 1961. 
be the symbols of all $t$ m.o.l. subs. No symbol $x_{i}$ appears in block $B$ or $C$ in any of the m.o.l.s., for all occurrences of $x_{i}$ in the $r$ rows or columns are in $D$ containing the m.o.l. subs. Any row contains each $x_{i}$ in each of the m.o.l.s.; since $B$ and $C$ contain no $x_{i}$, any row of $A$ contains each $x_{i}$ once in each of the $t$ m.o.l.s. The latin squares in $D$ being mutually orthogonal by hypothesis, no cell of $A$ can contain symbols $x_{i}$ in distinct latin squares. Hence $n-r \geqq t r$, proving Theorem 1.

TheORem 2. If a set of $t$ m.o.l.s. of order $n$ has a set of $t$ m.o.l. subs. of order $r$, with $r<n$, and there exists a latin square of order $n$ orthogonal to all $t$ m.o.l.s., then $r \leqq(n-r) /(t+1)+\left[r^{2} / n\right]$, the bracket denoting greatest integer.

(Note that the conclusion of Theorem 2 is stronger than that of Theorem 1 if and only if $r^{2} / n$ is not an integer.)

Proof. A transversal to a set of m.o.l.s. of order $n$ is a set of $n$ cells, one in each row, one in each column, and containing each symbol in each latin square. Thus a latin square orthogonal to each of the set of $t$ m.o.l.s. is equivalent to a set of $n$ transversals, no two having a common cell. $A, B, C, D ; x_{1}, \cdots, x_{r}$ denote the same as in the proof of Theorem 1. Let $T$ be a transversal; let $d$ be the number of cells of $D$ on $T$. In order that $T$ contain one cell in each row and column, $T$ contains $r-d$ cells in each of $B$ and $C$; in turn $T$ contains exactly $n-2(r-d)-d=n-2 r+d$ cells of $A$. Counting the $x_{i}$ separately for each of the m.o.l. subs., $T$ contains $t d$ symbols $x_{i}$ in $D$. As noted in proving Theorem $1, B$ and $C$ contain no $x_{i}$, and any cell of $A$ contains at most one symbol $x_{i}$ among all $t$ m.o.l.s. Thus $T$ contains at least $t r-t d$ cells of $A$. Hence $n-2 r+d \geqq t r-t d$. In turn $(t+1) d \geqq t r+2 r-n$, so that $d \geqq r-(n-r) /(t+1)$. The $n$ transversals without common cell include together all $r^{2}$ cells of $D$; since noninteger counts are excluded, some transversal $T$ of the set contains at most $\left[r^{2} / n\right]$ cells of $D$, so that $d \leqq\left[r^{2} / n\right]$ for this $T$. Hence $\left[r^{2} / n\right] \geqq r-(n-r) /(t+1)$, completing the proof.

The most interesting application of these theorems is given by the following

COROLlaRY. If a set of $r-1$ m.o.l.s. of order $n$ has a set of $r-1$ m.o.l. subs. of order $r$, with $r<n$, and there exists a latin square of order $n$ orthogonal to all $r-1$ m.o.l.s., then $n \geqq r^{2}$; if $n>r^{2}$, then $n \geqq r^{2}+r$.

Proof. Set $t=r-1$ in Theorems 1 and 2. The first part of the conclusion follows directly from Theorem 1 (with the last clause of hypothesis of the corollary superfluous). If $n>r^{2}$, then $\left[r^{2} / n\right]=0$, so 
that the conclusion of Theorem 2 is then $r \leqq(n-r) / r$; in turn $n \geqq r^{2}+r$.

A complete set of $n-1$ m.o.l.s. of order $n$ is equivalent to a projective plane of order $n$. Thus a set of $r-1 \mathrm{~m}$.o.l. subs. of order $r$ corresponds to a subplane of order $r$ if the set of m.o.l.s. of order $n$ is extended to a complete set. The Corollary has the same conclusion with weaker hypothesis than the following theorem attributed by M. Hall [6] to Bruck (and by Bruck to "folklore"): If a projective plane of finite order $n$ has a projective subplane of order $r$, with $r<n$, then $n \geqq r^{2}$; if $n>r^{2}$, then $n \geqq r^{2}+r$.

The author wishes to express thanks in print to his wife, Helen Meister Parker, for much assistance with typescripts and proofreading galley sheets.

\section{REFERENCES}

1. E. T. Parker, Orthogonal latin squares, Proc. Nat. Acad. Sci. U. S. A. 45 (1959), 859-862.

2. R. C. Bose, S. S. Shrikhande and E. T. Parker, Further results on the construction of mutually orthogonal latin squares and the falsity of Euler's conjecture, Canad. J. Math. 12 (1960), 189-203.

3. E. T. Parker, $A$ computer search for latin squares orthogonal to latin squares of order ten, Abstract 564-71, Notices Amer. Math. Soc. 6 (1959), 798.

4. Henry B. Mann, On orthogonal latin squares, Bull. Amer. Math. Soc. 50 (1944), 249-257.

5. R. T. Ostrowski and K. D. Van Duren, On a theorem of Mann on latin squares, Math. Comput. 15 (1961), 293-295.

6. Marshall Hall, Jr., Correction to "Uniqueness of the projective plane with 57 points," Proc. Amer. Math. Soc. 5 (1954), 994-997.

Remington Rand UNivaC Division of Sperry Rand Corporation and UNIVERSITY OF MINNESOTA 